\title{
Is it in a neonate's best interest to enter a randomised controlled trial?
}

Peter Allmark, Su Mason, A Bryan Gill and Christopher Megone University of Sheffield, University of Leeds, General Infirmary at Leeds and University of Leeds, respectively

\begin{abstract}
Clinicians are required to act in the best interest of neonates. However, it is not obvious that entry into a randomised controlled trial (RCT) is in a neonate's best interest because such trials often involve additional onerous procedures (such as intramuscular injections) in return for which the neonate receives unproven treatment or a placebo. On the other hand, neonatology needs to develop its evidence base, and RCTs are central to this task. The solution posited here is based on two points. First, "best interest" is not equivalent to "the best possible interest" only to "best interest within a certain realm". The realm of deliberation when asking the title question is the neonate's health. Deliberating in this realm may involve the exclusion from consideration of some factors that might be thought relevant (such as parental wealth). Furthermore, circumstances may dictate the need to deliberate on other factors that might be thought irrelevant (such as health care resources). Second, deciding on a neonate's best interest does not involve "putting oneself in its shoes". Rather, it involves asking in what it has an interest, or stake. These will include some things in which we all, as human beings, have a stake, such as medical progress. Putting these two points together, in the realm of health the answer to whether RCT entry is in a neonate's best interest is usually very finely balanced. Where this is the case, it is reasonable to invoke a broader notion of best interest and include a broader range of elements in which the neonate has a stake, including medical progress. In this way RCT entry can, usually, be said to be in a neonate's best interest.

(Fournal of Medical Ethics 2001;27:110-113)
\end{abstract}

Keywords: Neonates; randomised controlled trials; best interest; ethics

\section{Introduction}

This paper arose from discussions held at three colloquia under the auspices of "Euricon", a European Union funded project looking at informed consent to neonatal research trials. The aim of this paper is to show why the title question is of importance, why there is a problem, to analyse the term "best interest" and, finally, to suggest a possible answer based on a view of burdens, benefits and interests suggested by Feinberg. ${ }^{1}$ The paper is only concerned with research of direct benefit (so called, "therapeutic research").
Much of the discussion in this paper will be relevant to other areas of medicine, especially those concerned with incompetent patients. However, the focus on neonates could be justified in two ways. First, the need for research in neonatology seems particularly acute, being a relatively young discipline in which many of the treatments used are often only licensed for use in adults. Second, neonates raise the issue of best interest in a particularly pure form. There is no possibility that one can use a form of substituted judgment on the basis of the neonate's earlier preferences (something that can be done with some mentally ill adults). Neither may one foster a neonate's inchoate autonomy (as one may do with an older child).

\section{Why does the question matter?}

A "best interest principle" governs the conduct of parents and clinicians with regard to neonates receiving health care. ${ }^{2}$ Parents have the power of proxy consent but must use it only in their child's best interest. ${ }^{3}$ Clinicians are required to act in the best interest of incompetent patients ${ }^{4}$ (such as neonates) and of all patients (with the proviso that competent patients have given consent). Regarding research, the position in UK law appears to be that parents may consent to research of direct benefit on their neonate (only) if they are satisfied it is in its best interest, the same principle that governs consent to treatment.

It might be argued that therapeutic RCTs have a therapeutic aim, that as well as testing a new treatment, the new treatment is being given with the hope of benefit for the neonate, and that therefore, it is in the best interest of the neonate to be entered into such research. However, this line of argument is problematic for at least two reasons. First, the new treatment given as part of the RCT might not benefit the neonate, either because it has no effect or because it has deleterious effects that outweigh any benefit. If this wasn't a possibility then the RCT would be unnecessary. Thus, whilst we can say to parents of neonates receiving standard treatment that, on balance, the treatment is likely to benefit their child, we cannot say this with certainty to parents whose neonates are receiving new treatment within a trial. Second, as well as not benefiting the neonate, the new treatment might involve some burdens, such as additional and onerous interventions or some additional risk. Consider the following three simplified examples of RCTs. 
RCT 1 . There is a condition affecting neonates for which there is a standard treatment (A) and a new treatment $(B)$ on which there have been phase I and II trials that suggest it may improve on A. B is in the form of a regular intramuscular injection given in addition to A. A placebo controlled RCT is begun. B is only available through this RCT. Is it in the neonate's best interest to be entered into this trial? It would seem not. Any neonate entered stands a $50 \%$ chance of being burdened with a set of ineffective, placebo injections and a $50 \%$ chance of being burdened with a set of injections which may be beneficial, ineffective or deleterious. As such, we cannot say that, on balance, these injections are likely to help the neonate.

RCT 2. If we imagine that the trial is not placebo controlled, being instead a straight comparison of A with $\mathrm{A}$ plus $\mathrm{B}$, then a neonate stands a $50 \%$ chance of receiving exactly the same treatment as outside the trial and a $50 \%$ chance of receiving the burden (of intramuscular injections) in return for a treatment about whose efficacy in relation to established treatment we are unsure. Thus, again, it looks marginally against the neonate's best interest to be entered into the trial.

RCT 3. Lastly, imagine a trial which has minimal burdens. In such a case the neonate stands a 50/50 chance of receiving a treatment about whose efficacy we are unsure but without additional risk or other burden. An example of such an RCT might be one comparing two baby milk feeds for the preterm infant, where the mother and researcher are blinded to the allocation. An outcome measure of growth and development at two years would look at differences between the two. There would seem to be very little additional burden to the parents or the child. This form of trial has been done many times. Here the neonate seems to be as well off inside as outside the trial. Trial entry may plausibly be said to be in its best interest, it being one of two "best options" (ie standard treatment and treatment within the trial). It is not unreasonable to use the phrase, "Either of these two options would be best".

In summary, the problem is that many RCTs (ie those with any burdens) appear, on the face of it, to be contrary to a neonate's best interest. Yet, it is almost universally acknowledged that:

"Evidence based medicine is crucial for the effective management of patients. New-born babies and infants do not deserve to be treated on the basis of poorer quality evidence compared to older children and adults." 6

And RCTs play a central role in evidence based medicine. Let us turn, then, to an analysis of the term "best interest".

\section{Best interest}

The term "best interest" is most often used in legal and quasi-legal discussions (such as at case conferences) of the treatment of incompetent patients. Occasionally it may be invoked to discuss overriding the autonomy of competent patients (such as denying the full facts of diagnosis to seriously ill patients). ${ }^{7}$ Kopelman suggests there are three main uses of the term. ${ }^{8}$ The one that concerns us is its use as a marker for beneficence, the health carers' prima facie duty maximally to promote the good of the individual. ${ }^{9}$ It is this duty that underlies most health care interventions. Discussion of best interest in this form occurs most often where there is doubt as to what will promote the good of an incompetent individual (given that competent individuals are deemed capable of deciding for themselves). Such discussions occur in court where the doubt coincides with very serious consequences for the incompetent patient, as in cases where it is felt that it could be in someone's best interest to have life-saving treatment halted or not begun. ${ }^{10}$ Two points can be made about such discussions.

i) First, consideration of best interest may be limited to a certain realm of the child's life, for example, to his or her family relationships. As a result, certain factors that could be thought relevant to the child's overall good may be excluded. For example, in a custody case one would only consider whether the child is best placed with either mother or father, excluding consideration of whether she would be best placed somewhere else entirely. In the type of case we are considering the realm may be very broad. A clinician trying to decide what is in the best interest of a seriously handicapped neonate may need to include almost every aspect of that child's life and future. Certainly where such consideration of best interest occurs in court, as in cases involving discussion of the withdrawal or withholding of treatment from severely handicapped neonates, a very broad range of factors are included. ${ }^{11}$

However, perhaps not everything is relevant. For example, it may be thought wrong to include the relative prosperity of the parents in the deliberation, such that, of two identically handicapped neonates, the one with rich parents lives, the one with poor parents dies. ${ }^{12}$ The realm of the clinician's best interest deliberation is probably best described as being the neonate's health, something that is very broad but not all encompassing.

ii) Second, and on the other hand, consideration of a child's best interest seems to involve deliberation on factors other than those which can realistically be said to promote maximally the good health of the child. Resources are an obvious example; we may decide we cannot afford to give a child a remote chance of life because the treatment is too expensive. Parental wishes are another example. We might desist from doing something we think best for a child because parents do not consent to it. ${ }^{13}$ Thus, acting in a child's best interest is not necessarily doing what is ideal, only what is best in the circumstances. It does not follow that, say, when we consider whether we can afford a treatment for a child we are deliberating on its best interest. Rather, it 
means that prior to deliberation on its best interest we need to know the relevant circumstances.

It follows from these two points (i and ii) that deciding whether or not entry into RCTs is in a neonate's best interest will involve consideration of our realm of deliberation. Let us now turn to that task.

\section{Is RCT entry in a neonate's best interest?}

Feinberg offers a way of looking at burdens and benefits that may be helpful. ${ }^{14}$ For Feinberg a burden (or "harm") is something that frustrates, defeats or sets back our interests (and vice versa a benefit, presumably). An interest is something in which we have a stake, that is, we stand to lose or gain depending upon what happens to it. What is important here is that having an interest in something does not require being interested in it. For example, it will be contrary to a neonate's interest to be sterilised by a treatment even though the neonate doesn't himself care ${ }^{15}$ Having an interest does not even require that one be conscious or self-conscious. All living organisms have interests. A dog has an interest in eating unpoisoned food. A tree has an interest in receiving enough water and is harmed by a drought. Roughly, the interests of all living things are what they require in order to live well (as dog, tree or whatever). These interests will differ because a good life for a dog is different from a good life for a tree, similarly for a human.

On this account, deciding someone's best interest is not a matter of putting oneself in another's shoes and thinking what they would like, rather it is looking at the type of being the other is (ie a human being) and at the sort of things in which human beings have a stake (such as health and a good life). In this sense, the range of things in which neonates have a stake will be as broad as the range in which we all have a stake. This will mean not just absence of pain, but freedom from handicap, the development (and eventual use) of potentials to reason and of physical potentials, living in a peaceful and flourishing society, in fact, everything required to live a good human life. Included in this list will be the progress of science and medicine as this is something in which we all, as humans, have a stake.

If this is correct then we can say that the neonate has a stake in being entered in a RCT, that stake being the progress of medicine. How would having such a stake affect our attitude to whether or not RCT entry is in the particular neonate's best interest? We have already noted that the realm of consideration when we use the term "best interest" in this context is the neonate's health. Surely it is stretching credulity to suggest that the generic progress of medicine is an aspect of a neonate's health.

Whilst this seems correct, two points may counterbalance it. First, where we are deliberating between which of two options is in a neonate's best interest in terms of its health and find the point finely balanced, it seems reasonable to bring in other aspects of its life, to take a more global view of its best interest. Hence, if we cannot choose, on the basis of the neonate's health, between entry and non-entry to a RCT, the fact that the neonate, like all of us, has a stake in medical progress may swing the argument in favour of entry. Second, we have noted that other factors, such as resources, are relevant to our deliberation. Perhaps consideration of the progress of medicine (subsumed under the principle of justice) may swing us in favour of RCT entry where the balance between beneficence and non-maleficence alone does not suggest it.

In the three examples of RCTs above (RCT 1, 2 and 3 ) it is obvious that RCT 3 is very finely balanced indeed, hence it seems reasonable to include the neonate's stake in medical progress and conclude that RCT entry is in its best interest provided that other conditions are met, particularly, that there is parental consent. This is less clear with RCTs 1 and 2. With RCT 2 the balance also seems fairly fine; whilst half of the trial entrants will receive additional burdens there is some hope of benefit in return. And usually clinical equipoise will set the chance of benefit versus no benefit or deleterious effect at greater than $50 / 50$ (for example, on the basis of previous trials, phase I and II trials, or of plausible biological mechanisms). ${ }^{16}$ Thus, again, the global view of things in which the neonate has a stake may tip the balance in favour of saying that RCT entry is in its best interest.

\section{Most difficult case}

RCT 1 (in which the neonate may receive standard treatment plus placebo) presents the most difficult case. Can we say that it is finely balanced enough for global considerations to come into play when up to $50 \%$ of trial entrants are being exposed (for reasons of scientific rigour) to a burden in return for no possible health benefit, a placebo effect being very unlikely in a neonate? (NB: This does not imply that placebos should not be used in neonatal research.) Presumably it will depend on the burden and the view one takes of it. In many RCT 1 type trials the placebo will not be a great burden, for example, it may be an inert substance given through a pre-existing intravenous line. In such a case the health burdens and benefits do look finely balanced. As such, the global view of things again may tip the balance of the neonate's interest in favour of trial entry. ${ }^{17}$

With RCT 1 itself the placebo is given intramuscularly, something painful for the neonate, although usually this will not pose any more than minimal risk (for example, risk of death less than one per million, risk of major complication less than ten per million $\left.{ }^{18}\right)$. Consideration of the pain alone, however, may be enough for us to say, in health terms, that trial entry is not in the neonate's best interest and that, therefore, we should not go onto the next stage of bringing in global considerations to tip the balance in favour of entry. An alternative view might be that the pain involved is insignificant and that, therefore, the RCT entry versus non-entry 
options are finely balanced in terms of the neonate's (health) best interest. The frequency and duration of the intramuscular placebo injections may be important. The problem may be reduced in various ways. First, an alternative non-placebo trial design may be used. Second, the placebo could be given as a fake, intramuscular injection, where no needle actually penetrates the skin, although the rest of the ritual is followed (for example, by placing a smal plaster over the "injection site"). Third, anaesthetic cream might be applied prior to the injection. Any of these measures could make the health best interest considerations finely balanced and allow us to invoke the global considerations. A further, wider, consideration should perhaps be the trial organisation and the need for assurance of stringent safety monitoring being in place, such as a data monitoring and steering committees with a remit for tria safety, (as set out in the current Medical Research Council [MRC] guidelines for good clinical practice in clinical trials). ${ }^{19}$

\section{Conclusion}

So, is entry into therapeutic RCTs in the best interest of a neonate? It seems that, in terms of the neonate's health alone, the answer is finely balanced, but that when we invoke more global considerations of best interest, the answer is, usually, yes. Of these global considerations, the key one is the neonate's stake in the progress of medicine. However, invoking this consideration does not imply that it is reasonable to impose great burdens in terms of risk or suffering on the neonate for the sake of medicine.

"In research on man, the interest of science and society should never take precedence over considerations related to the well-being of the subject." ${ }^{20}$

What it does imply is that, where the balance between burdens and benefits in health terms is finely balanced between RCT entry and non-entry, it is reasonable to consider the neonate's stake in medical progress and, subject to parental consent, deem trial entry to be in its best interest.
Peter Allmark, MA, RN, is Nursing Lecturer, University of Sheffield. Su Mason, PhD, is Foint Operations Director, Northern \& Yorkshire Clinical Trials E Research Unit, University of Leeds. A Bryan Gill, MD, is Consultant Neonatologist, Peter Congdon Neonatal Unit, General Infirmary at Leeds. Christopher Megone, DPhil, is Senior Lecturer, Department of Philosophy, University of Leeds.

\section{References and notes}

1 Feinberg J. The moral limits of the criminal law: volume 1: harm to others. New York: OUP, 1984

2 It may also be termed the "welfare principle" or "acting in the neonate's interests".

3 Kennedy I, Grubb A. Medical law: text with materials [2nd ed]. London: Butterworths, 1994: 255

4 F v West Berkshire Health Authority [1989] 4 BMLR 1; Re F [1990] 2 AC 1.

5 See reference 3: 1052 and, for example, British Paediatric Association. Guidelines for the ethical conduct of medical research involving children. London: BPA, 1992.

6 Royal College of Paediatrics and Child Health. Safeguarding informed parental involvement in clinical research involving newborn babies and infants: a position statement. RCPCH: newborn babies 2 .

7 Hassan A, Hassan A. Do we always need to tell patients the truth? [letter]. Lancet 1998;352:1153.

8 Kopelman L. The best-interests standard as threshold, ideal and standard of reasonableness. Fournal of Medicine and Philosophy 1997;22:271-89.

9 Buchanan A, Brock D cited in reference 8: 277.

10 A key case involving a neonate was Re J (A minor) (Wardship: Medical Treatment) [1990] 3 All ER 930.

11 For example, Re J [1990] 3 All ER 930.

12 Arras J. Toward an ethic of ambiguity. Hastings Center Report 1984;Apr:25-33.

13 See reference 3: 1242

14 See reference $1: 31-51$.

15 We owe this point to $\mathrm{Mr}$ Christopher Coope.

16 Lilford R, Jackson J. Equipoise and the ethics of randomization. fournal of the Royal Society of Medicine 1995;88:552-9.

17 Deyn P, D'Hooge R. Placebos in clinical practice and research. fournal of Medical Ethics 1996;22:140-6.

18 Nicholson R. Medical research with children. Oxford: Oxford University Press, 1986: 119-20.

19 Medical Research Council. MRC guidelines for good clinical practice in clinical trials. London: MRC, 1998.

20 Declaration of Helsinki (adopted by the 18th Medical Assembly, Helsinki, Finland 1964 and last amended in South Africa, October 1996): III.4. 